\title{
Educação Física do Exame Nacional do Ensino Médio (Enem): uma revisão sistemática
}

\section{Physical Education in National Exam of Upper Secondary Education (Enem): a}

\section{systematic review}

\author{
José Pontes-Jr ${ }^{* * * *, 1}$, Leandro Sousa ${ }^{* * *}$, Evanildo Filho****, Kaio Oliveira***, Mark Carvalho ${ }^{* *}$ \\ *Universidade Estadual do Ceará, **Universidade Federal do Acre, ***Instituto Federal de Educação, Ciência e \\ Tecnologia do Ceará, ****Centro Universitário Católica de Quixadá
}

\begin{abstract}
Resumo
O estudo teve como objetivo avaliar as produções relativas à Educação Física no Enem por meio de uma revisão sistemática. Identificamos 13 trabalhos de 2010 a 2017 em diferentes bases de dados e analisados em 3 categorias: 1 ) aspectos conceituais das provas, 2) impactos políticos-curriculares do exame e 3) aspectos psicométricos das questões. Porém, todos mostraram evidências importantes sendo algumas positivas, como o fato de que o novo Enem trouxe mudanças no ensino da Educação Física e maior legitimidade à área, como também pontos negativos, como a superficialidade dos itens e déficits na estruturação dos mesmos.

Palavras chave: Avaliação educacional, Programas nacionais, Educação Física, Bibliometria
\end{abstract}

\begin{abstract}
The aim of this study was to evaluate the productions related to Physical Education in Enem through a systematic review. 13 papers from 2010 to 2017 in different databases were identified and analyzed in 3 categories: 1) conceptual aspects of the tests, 2) political-curricular impacts of the exam and 3) psychometric aspects of the questions. However, all of them showed important evidences being some positive ones, such as the fact that the new Enem brought changes in the teaching of Physical Education and greater legitimacy to the area, as well as negative points, such as the superficiality of the items and deficits in the structuring of them.

Keywords: Educational Assessment, National Programs, Physical Education, Bibliometrics
\end{abstract}

\section{Introdução}

A avaliação educacional no Brasil vem crescendo historicamente desde a década de 1990, dada à apreciação, nacional, do Sistema de Avaliação da Educação (SAEB), sendo uma ferramenta composta por avaliações em larga escala que têm como uns de seus objetivos: i) disponibilizar um diagnóstico sobre o contexto educacional brasileiro e ii) quais aspectos influenciam e interferem no desempenho dos alunos, sendo assim, material base da qualidade da educação do país. Ao final da década de 1990, ocorreu a criação do
Exame Nacional do Ensino Médio - ENEM pelo o Instituto Nacional de Estudos e Pesquisas Educacionais Anísio Teixeira - INEP, estritamente em 1998, o ENEM é instituído pela Portaria do Ministério da Educação MEC, $\mathrm{n}^{\circ} 438$ de maio de 1998 (Brasil, 2002). Dessa forma, o Exame é composto por quatros áreas do conhecimento e redação, sendo elas, i) Ciências da Natureza e suas Tecnologias, ii) Ciências Humanas e suas Tecnologias, iii) Matemática e suas Tecnologias, e iv) Linguagens, Códigos e e suas Tecnologias. Vale destaque, os itens de Educação Física estão inseridos nessa quarta área do conhecimento, sendo que esses itens foram incorporados apenas na Matriz de referência do Enem no ano de 2009 (Brasil, 2017).

Diante desse contexto, são muitas as inquietações acerca dos conteúdos de Educação Física exigidos no Enem, como por exemplo, a classificação dos itens de acordo com a taxonomia de Bloom (Pontes Jr, Sousa, \& Silva, 2015), os aspectos psicométricos dos itens relacionados aos conhecimentos de Esporte e Saúde no Enem (Pontes Jr et al., 2016), bem como que são de suma relevância para a comunidade científica e, principalmente, para as práticas pedagógicos nos diferentes contextos escolares.

Nessa perspectiva, se torna relevante à elaboração de uma síntese dos estudos que evidenciem as repercussões da inserção do componente curricular Educação Física como conteúdo na Matriz de referência do Enem, explicitando as principais evidências e definições de aspectos sobre as temáticas que estão sendo investigadas.

Com tal iniciativa, pretende-se contribuir no fomento de evidências a respeito do impacto dessa ação avaliativa nas práticas pedagógicas em Educação Física escolar, tanto entre os pesquisadores, quanto, principalmente, entre a comunidade escolar, em especial os professores de Educação Física. Diante do exposto, essa revisão sistemática teve como objetivo avaliar as produções relativas à Educação Física no Enem. 


\section{Métodos}

Trata-se de uma revisão sistemática acerca de produções científicas nacionais e internacionais publicadas no período de 2010 a 2017. Para isso foi realizada uma busca pareada nos seguintes bancos de dados: Google Acadêmico, SciELO, Biblioteca Digital Brasileira de Teses e Dissertações (BDTD), Latindex e em anais do Congresso Internacional em Avaliação Educacional. Em relação a esse Congresso, cabe indicar, foi feita essa opção por se tratar de um evento da área de Avaliação e que possui eixo temático específico de Avaliação da aprendizagem em Educação Física escolar.

Dessa forma foram utilizados os seguintes termos de busca com operadores booleanos "and" e "or": Avaliação, Evaluation, Assessment; Educação Física, Physical education; Larga escala, Large scale; Linguagens e códigos, Languages and codes; Enem; Exame Nacional do Ensino Médio; National Exam of Upper Secondary Education. Quando a esse último termo, tente-se como referência a página oficial em inglês do órgão brasileiro que organiza a exame, o INEP. Em seguida, foram definidas categorias com base na técnica de análise temática, pois possibilitaram a organização do material coletado em assuntos que os trabalhos pudessem ser discutidos em uma ou mais categorias, sendo: i) aspectos conceituais das provas; ii) aspectos psicométricos das questões e iii) impactos político-curriculares do exame.

A partir disso, foram compridas criteriosamente as seguintes etapas definidas: i) análise por título, ii) inclusão de trabalhos publicados no período de 2010 a 2017 , iii) leitura do resumo, iv) leitura do trabalho na íntegra e v) seleção e análise em pares dos artigos de acordo com os objetivos propostos.

A delimitação desse período se deu devido o componente curricular Educação Física ter sido inserido no Enem em 2009 e as publicações sobre a temática terem sido iniciadas no ano seguinte.

No entanto, vale ressaltar que foram excluídos estudos que i) se tratavam de outras revisões sistemáticas, ii) trabalhos de conclusão de curso de graduação e especialização, iii) resumos publicados em eventos científicos, e iv) estudos que não tinha relações com os aspectos/categorias de interesse desse estudo.

\section{Resultados}

Foram identificados 38 trabalhos que tinham alguma relação específica com o tema proposto, sendo excluídos 17 mediante a leitura dos resumos e 8 após a leitura na íntegra, sendo selecionados 13 trabalhos os quais atenderam aos objetivos do estudo.

Quanto às características das produções selecionadas, é possível identificar na Tabela 1, o nome dos autores, ano de publicação e os principais resultados dos estudos selecionados. Dessa maneira observam-se impactos da inserção de itens de Educação Física no Enem referente às práticas pedagógicas na escola, dificuldades na estruturação dos itens, bem como diversidade de conteúdos abordados no exame, possíveis desvantagens dos candidatos oriundos do Ensino Noturno, inserção de aulas teóricas desse componente curricular e maior potencial de valorização da área no contexto escolar.

Tabela 1.

Características das produções relativas à Educação Física no Enem a partir dos aspectos conceituais das provas, psicométricos das questões $e$ político-curriculares do exame.

\begin{tabular}{|c|c|c|}
\hline \multicolumn{2}{|c|}{ Aspecto } & Características \\
\hline & $\begin{array}{l}\text { Autor } \\
\text { (es)/ano }\end{array}$ & Principais resultados \\
\hline \multirow{5}{*}{$\frac{\mathscr{n}}{\stackrel{\Xi}{\Xi}}$} & $\begin{array}{l}\text { Franchi, } \\
\text { Ferreira, \& } \\
\text { Fagundes } \\
(2016)\end{array}$ & $\begin{array}{l}\text { Os conteúdos de maior frequência foram } \\
\text { dança estéticos e corpo, saúde e exercício } \\
\text { físico. }\end{array}$ \\
\hline & $\begin{array}{c}\text { Noaves } \\
(2015)\end{array}$ & $\begin{array}{l}\text { Para resolução dos itens, depende, } \\
\text { normalmente, da interpretação de textos, } \\
\text { ou seja, não requer um aprofundamento dos } \\
\text { conteúdos. }\end{array}$ \\
\hline & $\begin{array}{l}\text { Pontes Jr, } \\
\text { Sousa, \& } \\
\text { Silva }(2015)\end{array}$ & $\begin{array}{l}\text { Os itens de Educação Física do Novo Enem } \\
\text { (de } 2009 \text { a 2014) concentram-se na } \\
\text { categoria de "compreensão" na taxonomia } \\
\text { de Bloom. }\end{array}$ \\
\hline & $\begin{array}{l}\text { Souza Jr et al. } \\
\text { (2012) }\end{array}$ & $\begin{array}{l}\text { Maior frequência temática nos itens } \\
\text { relacionados ao conhecimento sobre o } \\
\text { corpo e atividades rítmicas e expressivas. }\end{array}$ \\
\hline & $\begin{array}{l}\text { Zaghi, } \\
\text { Simões, \& } \\
\text { Carbinatto } \\
(2015)\end{array}$ & $\begin{array}{l}\text { Os itens apresentaram discussões acerca de } \\
\text { diferentes esportes, porém não foi elencada } \\
\text { a possibilidade do conteúdo ginástica como } \\
\text { cultura e identidade social. }\end{array}$ \\
\hline \multirow{6}{*}{ 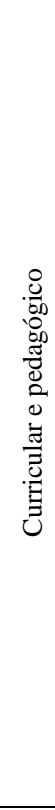 } & $\begin{array}{c}\text { Beltrão } \\
(2014)\end{array}$ & $\begin{array}{l}\text { Pressupõe-se que as práticas pedagógicas } \\
\text { dos professores de Educação Física } \\
\text { passaram a ser influenciadas pelo Enem. }\end{array}$ \\
\hline & $\begin{array}{l}\text { Fernandes, } \\
\text { Rodrigues e } \\
\text { Nardon } \\
\text { (2013) }\end{array}$ & $\begin{array}{l}\text { Há certas desvantagens dos alunos que } \\
\text { estudam no período noturno, pois seu } \\
\text { processo formativo é interferido, o que } \\
\text { contribui para o baixo desempenho no } \\
\text { Educação Física no Enem. }\end{array}$ \\
\hline & Kohl (2010) & $\begin{array}{l}\text { O novo Enem pode provocar } \\
\text { "desequilíbrios pedagógicos" quanto aos } \\
\text { seus objetivos de ensino e oportunidade de } \\
\text { legitimação da Educação Física no âmbito } \\
\text { escolar. }\end{array}$ \\
\hline & Santos (2014) & $\begin{array}{l}\text { A disciplina no Enem contribuiu para que o } \\
\text { ensino na escola fosse além das práticas } \\
\text { corporais, contemplando aspectos } \\
\text { cognitivos da área. }\end{array}$ \\
\hline & Silva (2013) & $\begin{array}{l}\text { Não foram evidenciadas alterações } \\
\text { significativas na prática pedagógica do } \\
\text { professor devido à participação no da } \\
\text { Educação Física no Enem }\end{array}$ \\
\hline & $\begin{array}{l}\text { Sousa, Sobral } \\
\text { e Trompieri } \\
\text { Filho }(2015)\end{array}$ & $\begin{array}{l}\text { Percebe-se alterações nas práticas } \\
\text { pedagógicas, principalmente no que diz } \\
\text { respeito o teor teórico empregado nas } \\
\text { aulas. }\end{array}$ \\
\hline \multirow[b]{2}{*}{ 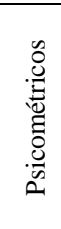 } & Sousa (2017) & $\begin{array}{l}\text { Os itens de } 2014 \text { apresentaram baixo índice } \\
\text { de discriminação. }\end{array}$ \\
\hline & $\begin{array}{l}\text { Pontes Jr et al. } \\
\quad(2016)\end{array}$ & $\begin{array}{l}\text { Foram identificados inadequada } \\
\text { consistência interna dos itens, nível fácil ou } \\
\text { moderado das questões e melhor } \\
\text { desempenho das escolas privadas, região } \\
\text { sul e sudeste. }\end{array}$ \\
\hline
\end{tabular}

Fonte: Próprios autores. 


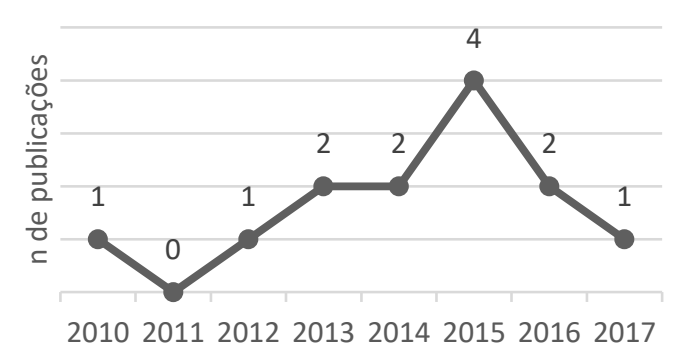

Ano das publicações

Figure 1. Frequências/números de publicação por ano. Fonte: Próprios autores

A figura 1 apresenta o quantitativo de trabalhos selecionados durante o período de 2010 a 2017. Desse modo, foram identificadas ascendências de produções de 2012 a 2015 em que mais da metade $(53,8 \%)$ das produções foram realizadas nesse período.

\section{Discussão}

A partir da busca nos dispositivos que retém os dados, foi possível mostrar uma ascendência de produções de 2012 a 2015 e descendência nos dois anos posteriores (2016 e 2017), como também, foram evidenciados impactos positivos da inserção de itens de Educação Física no Enem referente às práticas pedagógicas na escola, além da dificuldade na elaboração de itens, entre outros. Diante disso, notam-se apontamentos que carecem respostas consolidadas, nesse sentido, será o centro das discussões nos parágrafos seguintes.

Desse modo, no que diz respeito ao conhecimento de conteúdos de Educação Física nos Itens do Enem, foi evidenciado prevalência de itens relacionados às atividades rítmicas e expressivas e conhecimento sobre o corpo nas edições de 2009 a 2013 (Pontes Jr et al., 2016), o que induz que há uma certa preocupação de avaliar os estudantes na vertente teórica, através de conhecimentos pertinentes que podem contribuir para prática. Ainda esses autores, constataram que os itens relacionados aos conhecimentos de esportes e saúde precisam ser aprimorados.

Diante disso, ressalta-se a importância de conhecimento sobre o corpo nas aulas de Educação Física, especialmente no Ensino Médio, em que possibilita ao professor discutir com os alunos sobre diferentes temas presente nesse conteúdo, como por exemplo, anatomia e aspectos fisiológicos relacionados à atividade física (Costa \& Silva, 2014), além do mais, há a possibilidade de ser um dos conteúdos exigidos no Enem. Nessa perspectiva, Gutierrez et al. (2014) obtiveram resultados considerados positivos na aprendizagem dos alunos ao abordar essas temáticas na escola.

Do mesmo modo, o estudo considera que as atividades rítmicas e expressivas, quando trabalhadas por meio de vivências através de danças e de outras experiências consideradas exitosas, como o teatro, são práticas relevantes na ampliação das possibilidades da expressão corporal e cultura corporal do movimento, sendo estas, umas das principais propostas da Educação Física enquanto disciplina na Educação Básica (Silva, Pizani, Mendes, \& Barbosa-Rinaldi, 2015).

Diante dessas e de outras possibilidades de ensino da Educação Física na Educação Básica, uma vez que vêm sendo exigidos conhecimentos dos alunos no Enem, embora com poucas exigências quanto ao grau de dificuldades (Novaes, 2015), compete aos professores responsáveis por essa disciplina proporcionar experiências exitosas, partindo-se da valorização de certos conteúdos e metodologias fundamentais na formação dos alunos.

Dessa forma, do ponto de vista curricular e pedagógico da Educação Básica, o Enem potencializa alterações na Educação Física como no disciplina escolar, com perspectivas a influenciar o próprio currículo, pois a robustez que esse sistema tem ganhado no Brasil por ser uma avaliação recente, de maneira natural introduz mudanças, portanto, busca-se brio na qualidade da educação advinda (Beltrão, 2014). Em corroboração, Sousa, Sobral e Trompieri (2015) apontam que as avaliações em larga escala, tratando-se do Enem, pode influenciar na prática docente.

Por esse lado, foi perceptível no estudo de Costa e Rodrigues (2015) que ao analisar as orientações, diretrizes e referenciais curriculares relacionados ao ensino da disciplina de Educação Física no Ensino Médio do Estado do Maranhão, identificaram no documento referente o planejamento anual de atividades curriculares e extracurriculares dessa disciplina que a escolha do conteúdo deve levar em consideração tanto as especificidades locais e regionais, quanto aos exames seletivos da região e o Enem.

Em consonância, Guedes et al. (2017) identificaram que a grade curricular de uma escola de Ensino Médio profissionalizante tem como prioridade o Enem. Diante disso, estes estudos mostram a preocupação da escola com as práticas pedagógicas dessa disciplina a partir de exigências externas, como no caso do Enem.

Diante disso, as alterações nos currículos das escolas se mostram como pontos positivos e nova postura adotada pelos docentes a fim melhorar a qualidade da Educação.

Esses pontos supracitados das mudanças da Educação Física na Educação Básica potencializam resultados melhores no Enem sendo uma avaliação estritamente brasileira como em outras áreas, visto que no estudo da OCDE (2016), organização responsável pelo PISA (Programa Internacional de Avaliação de Estudantes de 15 anos de diversos países), indica que atividades extracurriculares de fins culturais e esportes tendem a contribuir com a melhoria do desempenho cognitivo e social dos alunos.

É oportuno relatar que, assim como foi evidenciado déficits nos itens de Educação Física no Enem (Pontes Jr et al., 2016), também foram encontradas limitações no PISA (Fernandez-Cano, 2016), apesar de nesse não possuir questões de Educação Física, mas como se trata de uma avaliação externa de larga escala e aplicada em diferentes contextos, cabe indicar que é possível que ocorram problemas que se ajustam como um maior tempo de aplicação (o Enem é do final da década de 1990 e o PISA teve sua primeira edição em 2000). Com isso, 
demonstra que, embora não se permita erros exagerados na elaboração de exames, é comum encontrar falhas que podem influenciar nos resultados do que se pretende examinar. Sendo assim, é preciso maior cautela e rigor metodológico na elaboração desses exames.

Contudo, há diferentes objetos/fatores que podem influenciar direta ou indiretamente no desempenho dos alunos. Com isso, a partir das produções referentes à temática aqui estudada, facilita o conhecimento sobre esses mecanismos, além de tornar evidente quais são os estudos que estão sendo desenvolvidos acerca desse sistema de avaliação relativamente novo no Brasil, bem como as suas eventuais contribuições para comunidade científica e escolar.

\section{Conclusão}

Conclui-se que há diferentes produções científicas acerca da inserção dos conteúdos de Educação Física no Enem, no entanto, nos três aspectos definidos, pode-se apontar uma maior produção voltada para discussões dos aspectos conceituais das provas, em seguida dos impactos políticos-curriculares do exame e por últimos aspectos psicométricos das questões. Porém, todos mostraram evidências importantes sendo algumas positivas, como o fato que novo Enem trouxe mudanças no ensino da Educação Física e maior legitimidade da área, como também pontos negativos, no caso da superficialidade dos itens e déficits na estruturação dos mesmos.

A partir desse estudo esperam-se estimular mudanças nas práticas pedagógicas e impulsionar ou instigar os professores, gestores bem como órgãos governamentais a discutir e rever alguns impasses que merecem uma atenção detalhada. Especificamente, o artigo propõe o aprimoramento das provas elaboradas e sugere alternativas à inserção da Educação Física na área de Linguagens e Códigos. Por fim, acreditamos que o estudo também pode contribuir com a fundamentação teórico-metodológica e político da Educação Física no ENEM.

\section{Agradecimentos}

José Pontes-Jr ${ }^{1}$ : Bolsa PNPD da CAPES.

\section{Referências}

Beltrão, J. A. (2014). A educação física na escola do vestibular: as possíveis implicações do ENEM. Movimento, revista de Educação Física da UFRGS, 20(2):819-840. Disponível em: <http://www.seer.ufrgs.br/index.php/Movimento/artic le/view/41801/28928>. Acesso em: 14 de julho de 2017.

Brasil. (2002) Ministério da Educação. Instituto Nacional de Estudos e Pesquisas Educacionais Anísio Teixeira. Enem: Documento Básico. Brasília: MEC/Inep. Disponível em: < http://portal.inep.gov.br/documents/186968/484421/E $\mathrm{NEM+-+Exame+Nacional+do+Ensino+M \% C3 \% A9di}$ o+documento+b\%C3\%A1 sico+2002/193b6522-cd524ed2-a30f-24c582ae941d?version=1.2>. Acesso em: 13 de julho de 2017.
Brasil. Instituto Nacional de Estudos e Pesquisas Educacionais Anísio Teixeira - Inep. (2017). Novo ENEM. Disponível em: <http://portal.mec.gov.br/busca-geral/179-estudantes108009469/vestibulares-1723538374/13318-novo-ene m>. Acesso em: 12 de julho de 2017.

Costa, F. S., \& Rodrigues, J. P. (2015). A educação física escolar e o currículo no ensino médio caxiense. Revista Educação - UNG, 10(1):43-51. Disponível em:<http://revistas.ung.br/index.php/educacao/article/ view/2061/1564>. Acesso em 13 de Jul. 2017.

Costa, N. T. M., \& Silva, A. C. (2014). Corpo e educação física escolar no ensino médio: a visão dos alunos. Revista Brasileira de Ciência do Esporte, 36(2):223-237. Disponível em: < http://revista.cbce.org.br/index.php/RBCE/article/dow nload/2129/1087>. Acesso em: 13 de julho de 2017.

Fernandes, A., Rodrigues, H. A., Nardon, T. A. (2013). A inserção dos conteúdos de educação física no enem: entre a valorização do componente curricular e as contradições da democracia. Motrivivência, 25(40): 13-24.

http://dx.doi.org/10.5007/2175-8042.2013v25n40p13

Fernandez-Cano, A. (2016). A Methodological Critique of the PISA Evaluations. RELIEVE, 22(1):1-16. Doi:http://dx.doi.org/10.7203/relieve.22.1.8806.

Franchi, S. (2016). Educação física no Enem: valorização da cultura corporal. Em anais do VI Seminário Nacional de Pesquisa em Educação. Disponível em: <http://online.unisc.br/acadnet/anais/index.php/seped u/article/view/14908/345>. Acesso em: 13 de julho de 2017.

Guedes, J. D., Duarte, C. C. F. Feitosa, J. G., Santos, L. G. F.; Silva, P. A. B. Souza, A. J. C. A, Vieira, T. A. (2017). Escola Profissionalizante e o método de ensino e aprendizagem na Educação Física. Id on line Multiciplinary and Psycology Journal, 10(33):87-93, 2017. http://dx.doi.org/10.14295/idonline.v10i33.640.

Gutierrez, M. V. B., Dias, D. V., Andrade, J. C. T., \& Daré, L. R. (2014). Descobrindo o corpo humano: difundindo o conhecimento de anatomia humana nas escolas públicas de Uruguaiana/RS. Anais do Salão Internacional de Ensino. Pesquisa e Extensão, 6(3). Disponível em: <http://publicase.unipampa.edu.br/index.php/siepe/art Icle/view/7493\&gt>. Acessado em: 12 de abr. 2017.

Kohl, H. G. (2010). Educação física na educação básica e o novo enem: novos desafios e novas possibilidades. Em IV anais do Encontro de ensino, pesquisa e extensão da faculdade Senac, 27 e 28 de outubro. Disponível em:

http://www.faculdadesenacpe.edu.br/encontro-de-ensi no-pesquisa/2011/IV/anais/comunicacao/022_2010_a p_oral.pdf $>$. Acesso em: 12 de julho de 2017.

Machado, S. S. G. (2015). A educação física no exame nacional do ensino médio. Trabalho de Conclusão de Curso. Universidade Estadual da Paraíba. Centro de Ciências Biológicas e da Saúde, Paraíba, Brasil. Disponível em:

http://dspace.bc.uepb.edu.br/jspui/bitstream/12345678 9/8650/1/PDF\%20-\%20Su\%C3\%AAnia\%20de\%20S 
ousa\%20Gouveia\%20Machado.pdf>. Acesso em: 13 de julho de 2017.

Novaes, R. C. (2015). A educação física no exame nacional do ensino médio. Dissertação (Mestrado). Universidade do Estado do Rio de Janeiro. Centro de Educação e Humanidades. Instituto de Educação Física e Desportos, Rio de Janeiro, Brasil.

OECD. (2016). Low Performing Students: Why They Fall Behind and How to Help Them Succeed, PISA, OECD Publishing, Paris, http://dx.doi.org/10.1787/9789264250246-en.

Pereira, A. I. (2015). A educação física: análise dos conteúdos das questões de educação física nas provas do enem de 2009 - 2012. Trabalho de Conclusão de Curso. Universidade Estadual Paulista - Júlio de Mesquita Filho. Instituto de Biociências, Rio Claro, São Paulo, Brasil. Disponível em: < https://repositorio.unesp.br/bitstream/handle/11449/13 1750/000853548.pdf? sequence $=1$ \&is Allowed $=y>$. Acesso em: 13 de julho de 2017.

Pontes Jr, J. A. F., Silva, A. G., Tavares, E. D., Sousa, L. A., Bastos, F. A. C., Cruz, F. N. I., Almeida, L. S. (2016). Aspectos psicométricos dos itens de Educação Física relacionados aos conhecimentos de Esporte e Saúde no Exame Nacional do Ensino Médio (enem). Revista motricidade, 12(s1):12-21. Doi: http://dx.doi.org/10.6063/motricidade.8482.

Pontes Jr, J. A. F., Sousa, L. S., \& Silva, A. G. (2015). Itens de educação física no Exame Nacional do Ensino Médio (Enem) via taxonomia de Bloom. Em Anais do VI Congresso Internacional em Avaliação Internacional, pp. 1626-1643, Fortaleza-CE, Brasil. Disponível em:

http://www.nave.ufc.br/vi_ciae/views/documentos/VI \%20CONGRESSO \%20INTERNACIONAL\%20EM $\% 20$ AVALIA $\%$ C3\%87\%C3\%830\%20EDUCACION AL_29\%20OUTUBRO\%202015.pdf>. Acesso em: 14 de julho de 2017.

Santos, K. (2014). Os saberes da educação física em pauta: conteúdos de ensino da educação básica $e$ conhecimentos constituintes dos processos de seleção para ingresso no ensino superior. Dissertação (Mestrado). Universidade Estadual do Centro-Oeste, (UNICENTRO), Guarapuava, PR, Brasil. Disponível em:

http://tede.unicentro.br:8080/jspui/bitstream/tede/355/ 1/PR\%20KATIANE\%20DOS\%20SANTOS.pdf $>$.

Acesso em: 13 de julho de 2017.

Silva, D. O., Pizani, J., Mendes, E. H., \& Barbosa-Rinaldi, I. P. (2015). As disciplinas rítmicas e dançantes nos cursos de licenciatura em Educação Física do estado do Paraná. Pensar a Prática, 18(3):530-543. https://doi.org/10.5216/rpp.v18i3.27651.

Silva, R. V. C. (2013). Educação física: identidade, saberes e prática pedagógica após a participação da disciplina no exame nacional do ensino médio. Dissertação (Mestrado). Universidade Tuiuti do Paraná, Programa de Pós-Graduação em Educação, Curitiba, Brasil. Disponível em: < http://tede.utp.br:8080/jspui/bitstream/tede/590/1/ED UCACAO\%20FISICA\%20IDENTIDADE\%20SABE
RES\%20E\%20PRATICA.pdf>. Acesso em: 13 de julho de 2017.

Sousa, L. A., Sobral, A. E. B., \& Trompieri Filho, N. (2015). Educação física em avaliações em larga escala: o caso do exame nacional do ensino médio - enem. Revista Educação e Linguagem, 2(1):60-71. Disponível em: < http://www.fvj.br/revista/wp-content/uploads/2015/10 15_Educ_2015_1.pdf>. Acesso em: 13 de julho de 2017.

Sousa, L. A. (2017). Análise psicométrica dos itens de educação física do exame nacional do ensino médio (ENEM) via teoria clássica dos testes. Dissertação (Mestrado). Universidade Federal do Ceará, Programa de Pós-graduação em Educação Brasileira, Fortaleza, CE, Brasil. Disponível em: < http://www.repositorio.ufc.br/bitstream/riufc/22251/1/ 2017_dis_lasousa.pdf $>$. Acesso em: 13 de julho de 2017.

Souza Júnior, O. M., Diniz, I. K. S., Ditomaso, A., \& Darido, S. C. (2012). Educação física no enem: análise das questões à luz dos pcns. CPQMH / II Congresso Internacional de Educação Física Esporte e Lazer CIEFEL / VI Shoto Workshop, pp. 345-355.

Zaghi, F., Simões, R. M. R., \& Carbinatto, M. V. (2015). Ginástica e exame nacional do ensino médio. Conexões: revista da Faculdade de Educação Física da UNICAMP, Campinas 13:115-126. Disponível em: $<$ https://periodicos.sbu.unicamp.br/ojs/index.php/cone xoes/article/download/8637579/5270>. Acesso em: 13 de julho de 2017.

Zaghi, F. H. L. S. (2014). Educação física escolar e a prova do ENEM: convergências e divergências. Dissertação (Mestrado). Universidade Federal do Triângulo Mineiro, Uberaba. Minais Gerais, Brasil. Disponível em: http://bdtd.uftm.edu.br/bitstream/tede/323/5/Dissert\% 20Flavio\%20H\%20L\%20S\%20Zaghi.pdf>. Acesso em: 12 de julho de 2017. 